\title{
An Unusual Case of Placenta Abruption Leading to Couvelaire Uterus in a Previable Pregnancy
}

\author{
Goh Siak Ming ${ }^{a}$, b, Wai Kheong Ryan Lee ${ }^{a}$, Shu Qi Tan ${ }^{\mathrm{a}}$
}

\begin{abstract}
Couvelaire uterus or uteroplacental apoplexy is a rare complication of abruptio placentae whose etiology is unknown. It is a clinical diagnosis made during visual inspection of the uterus. Immediate management is usually conservative and hysterectomy is usually not required. Our case highlights the importance of early recognition of a clinically unstable previable pregnant patient with suspected placenta abruption and early surgical recourse with successful emergency hysterotomy.
\end{abstract}

Keywords: Placenta abruption; Couvelaire uterus; Previable pregnancy

\section{Introduction}

Placenta abruption is a clinical diagnosis. Its presentation below 24 weeks is rare, but it poses a difficult clinical dilemma as immediate delivery is usually indicated in a previable pregnancy. We present a case of a clinically unstable previable pregnant patient with suspected placenta abruption and Couvelaire uterus. This is an uncommon presentation found during visual inspection of the uterus that has dark purple patches with ecchymosis.

A Couvelaire uterus does not affect the uterine ability to contract and decompression usually allows constriction of spiral arteries to achieve hemostasis. A hysterectomy may be indicated as a life-saving measure if hemostasis cannot be achieved adequately in view of disseminated intravascular coagulopathy. Our case highlights the importance of early recognition of a clinically unstable previable pregnant patient with suspected abruption and early surgical recourse with emergency hysterotomy for life-saving measures.

Manuscript submitted March 31, 2020, accepted April 7, 2020

aDepartment of Obstetrics and Gynaecology, KK Women and Children's Hospital, 100 Bukit Timah Road, Singapore 229899, Singapore

${ }^{b}$ Corresponding Author: Goh Siak Ming, Department of Obstetrics and Gynaecology, KK Women and Children's Hospital, 100 Bukit Timah Road, Singapore 229899, Singapore. Email: siakming@gmail.com

doi: https://doi.org/10.14740/jmc3462

\section{Case Report}

A 37-year-old woman, gravida 2 para 1, presented to a tertiary women's hospital at 21 weeks gestation in her second pregnancy complaining of vaginal bleeding of 1-day duration changing three sanitary pads and colicky lower abdominal cramps. History taking revealed she had one previous full-term normal vaginal delivery that was complicated by pregnancyinduced hypertension 2 years earlier. She booked late at 19 weeks in her second pregnancy and was started on aspirin for this pregnancy to reduce the risk of preeclampsia. Antenatal bloods were normal. She declined maternal serum screening for chromosomal aneuploidies. Fetal anomaly screening at 20 weeks showed low risk with no fetal anomalies except for a low-lying anterior mid placenta.

Her vital signs showed a temperature of $36.7^{\circ} \mathrm{C}$ and maternal tachycardia of 107 beats per minute with blood pressure reading of 136/95. On examination, her abdomen was soft and the uterus was relaxed and not woody. Speculum examination showed no active bleeding with cervical os closed and a small clot evacuated. Her reflexes were normal with no signs of clonus suggestive of impending eclampsia. Fetal heart measured was normal at 147 beats per minute. Ultrasound scan showed a low-lying anterior placenta. The clinical diagnosis then was threatened miscarraige and low-lying placenta.

Subsequently, she was admitted in hospital for further monitoring. Initial blood tests revealed a hemoglobin of 10.7 $\mathrm{g} / \mathrm{dL}$, low platelet of $90 \times 10^{9} / \mathrm{L}$, deranged coagulation profile and mild acute kidney impairment with raised creatinine of $99 \mathrm{mmol} / \mathrm{L}$. She was transferred to the high dependency unit for further management and was reviewed $4 \mathrm{~h}$ later when she suddenly complained of increasing vaginal bleeding and worsening generalized lower abdominal pain. Vital signs revealed blood pressure reading of $108 / 61 \mathrm{~mm} \mathrm{Hg}$ with persistent maternal tachycardia at 110 beats per minute. On physical examination, the uterus was now tense and woody hard and vaginal examination using speculum revealed the cervical os was closed with active vaginal bleeding and clots evacuated. Atrans-abdominal ultrasound showed a heterogenous area anterior to the placenta suggestive of blood products with placenta abruption (Fig. 1).

A repeat set of blood tests performed immediately showed a worsening drop in platelet levels from $90 \times 10^{9} / \mathrm{L}$ to $53 \times$ $10^{9} / \mathrm{L}$ and a drop in hemoglobin from 10.7 to $7.8 \mathrm{~g} / \mathrm{dL}$. Coagulation profile remained severely deranged. The clinical di- 


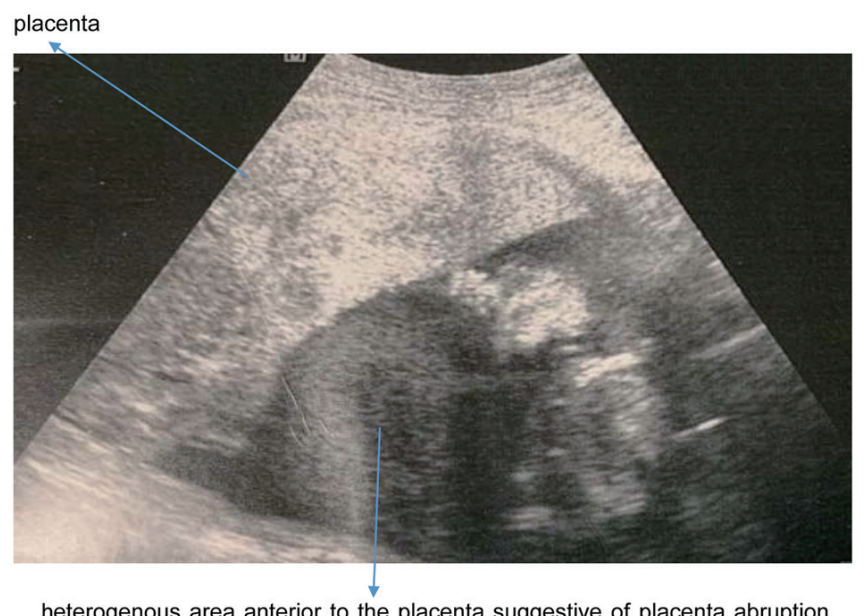

heterogenous area anterior to the placenta suggestive of placenta abruption with blood products

Figure 1. A heterogenous area anterior to the placenta suggestive of placenta abruption with blood products.

agnosis was consumptive coagulopathy secondary to placenta abruption. In view of the patient's deteriorating condition, the patient subsequently underwent explorative laparotomy keep in view hysterotomy at 21 weeks gestation.

Under general anesthesia, a lower segment hysterotomy was performed via Pfannenstiel incision. Intraoperative findings revealed a Couvelaire uterus with clots noted on incision into uterus. The fetus and an entirely separated placenta were delivered complete. In total, there was estimated blood loss of $1,000 \mathrm{~mL}$. The appearance of the uterus was consistent with Couvelaire uterus (Fig. 2). The uterus was closed in two layers and hemostasis was achieved. The patient recovered well subsequently with blood products replacement and her coagulation profile was normal after with post-operative hemoglobin of $9.2 \mathrm{~g} / \mathrm{dL}$. She was discharged well 3 days later. Histopathology of placenta showed retroplacental and retromembranous hemorrhage with intervillous hemorrhage consistent with placental abruption.

\section{Discussion}

We present an unusual case of placental abruption at previable gestation of $<24$ weeks with Couvelaire uterus during laparotomy. This is an uncommon presentation with clinical diagnosis made during inspection with the uterus found to have dark purple patches with ecchymosis. Couvelaire or uteroplacental apoplexy was first described in 1911 [1]. It is a rare non-fatal complication of severe abruption and is estimated to complicate $5 \%$ of all cases of abruption [2]. This entity is often underreported and the incidence is difficult to estimate because the diagnosis is made by direct visualisation or biopsy.

Couvelaire uterus is caused when hemorrhage from placental blood vessels seeps into decidua basalis causing placental separation, followed by infiltration in the lateral portions of the uterus [3]. However, this does not usually affect the uterine ability to contract and surgical decompression usually allows constriction of spiral arteries [2]. Placenta abruption is an ob-

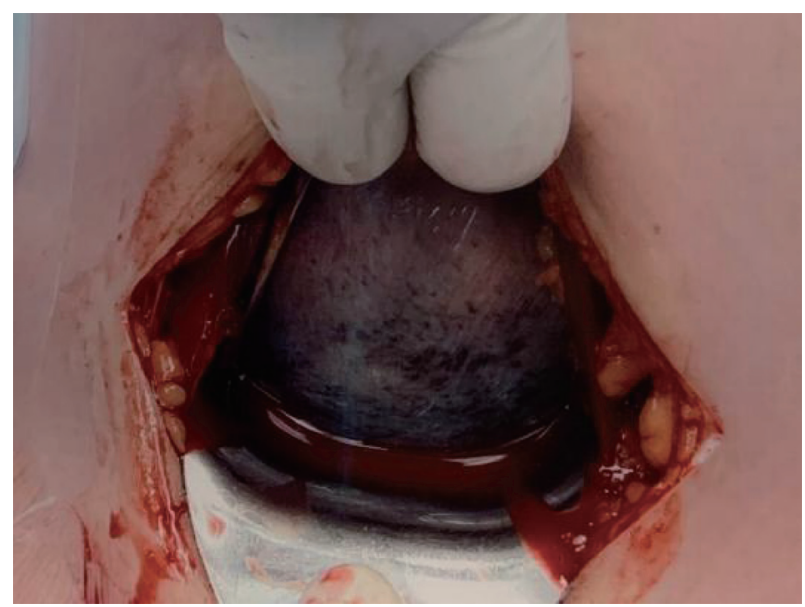

Figure 2. Intraoperative findings during laparotomy with dark purple patches of the uterus suggestive of Couvelaire uterus.

stetric emergency associated with increased maternal and fetal morbidity and mortality. Placental abruption involves separation of the normally situated placenta after the 20th week of gestation and prior to birth. Despite its clinical significance, there are no reliable diagnostic tests or biomarkers to predict or prevent the occurrence of abruption [4].

The diagnosis of placenta abruption is often a clinical one with a low reported accuracy of ultrasound-based diagnosis to be $<30 \%$ [5]. Despite this, ultrasound should be performed to exclude other causes of vaginal bleeding including placenta previa or vasa previa. Classic echographia signs including peripheral detachment of the placenta or increased thickness associated with a variably echogenic zone depending on hematoma are likely diagnostic of placenta abruption [6].

Placenta abruption can present with painful or painless vaginal bleeding accompanied by uterine contractions and a non-reassuring fetal heart rate pattern. The exact etiology of abruption is unknown but risk factors include advanced maternal age, low social economic status, cigarette smoking, trauma, cocaine, maternal hypertension, polyhydramnios, multiple pregnancy, thrombophilia and previous history of abruption [7, 8]. Our patient does have a history of chronic hypertension that increases her risk of placenta abruption. It is suggested that placenta abruption involves placental or vascular abnormalities because of failure of secondary invasion of trophoblastic villi. Abnormal placentation, vascular malformations, and increased fragility of vessels predispose to hematoma formation, resulting in separation of the placenta [9].

The management of placenta abruption with Couvelaire uterus depends on the clinical status of the patient. There have been cases of successful conservative management of Couvelaire uterus [10]. A Couvelaire uterus does not affect the uterine ability to contract and decompression usually allows constriction of spiral arteries to achieve hemostasis. However, in cases with acute clinical deterioration such as an acute abdomen or coagulopathy, early recourse to surgical intervention is paramount.

The surgical management of Couvelaire uterus is by hysterotomy or hysterectomy. Hysterectomy can be considered 
when there is profound myometrial damage or uncontrolled bleeding despite conservative measures by hemostatic brace sutures or uterotonics. In our case, decision was made for hysterotomy in view of an acute abdomen in a previable pregnancy $<24$ weeks with clinical suspicion of placenta abruption. The patient was counselled that the fetus at 22 weeks of gestation with suspected placenta abruption had no chance of survival and that an explorative laparotomy with hysterotomy was necessary and a hysterectomy may be performed as life saving measure despite conservative measures to arrest torrential bleeding that can lead to disseminated intravascular coagulopathy, multi-organ failure and death.

Our patient was discharged well on follow up. She was also counselled extensively on the future implications of placenta abruption leading to hysterotomy. This includes increased risk of placenta abruption, placenta previa, and uterine rupture in her next pregnancy, and she was advised to avoid pregnancy for at least 1 year with contraceptive advice given.

In summary, Couvelaire uterus or uteroplacental apoplexy is a rare complication of abruptio placentae whose etiology is unknown. It is a clinical diagnosis made on visual inspection of the uterus. Its immediate management is usually conservative and hysterectomy is usually not required. Our case highlights the importance of early recognition of a clinically unstable previable pregnant patient with suspected abruption and early surgical recourse with emergency hysterotomy for life-saving measures.

\section{Acknowledgments}

None to declare.

\section{Financial Disclosure}

None to declare.

\section{Conflict of Interest}

None to declare.

\section{Informed Consent}

Informed consent has been obtained for this paper.

\section{Author Contributions}

All authors contributed equally to writing the case report and discussion around the case.

\section{Data Availability}

The authors declare that data supporting the findings of this study are available within the article.

\section{References}

1. Couvelaire A. Deux nouvelles observations d'apoplexie utero-placentaire hemorrhagies retro-placentaires avec infiltration sanguine de la pavoi musculaire de l'uterus. Ann Gynecol Obstet. 1912;9:486.

2. Habek D, Selthofer R, Kulas T. Uteroplacental apoplexy (Couvelaire syndrome). Wien Klin Wochenschr. 2008;120(3-4):88.

3. Hubbard JL, Hosmer SB. Couvelaire uterus. J Am Osteopath Assoc. 1997;97(9):536-537.

4. Abasi J, Jeremiah I, Ekine AA. Risk factors and pregnancy outcome of placental abruption at the Niger delta university teaching hospital, okolobiri, south-south. Nigeria Br J Med Res. 2015;5:1000-1100.

5. Glantz C, Purnell L. Clinical utility of sonography in the diagnosis and treatment of placental abruption. J Ultrasound Med. 2002;21(8):837-840.

6. Shinde GR, Vaswani BP, Patange RP, Laddad MM, Bhosale RB. Diagnostic performance of ultrasonography for detection of abruption and its clinical correlation and maternal and foetal outcome. J Clin Diagn Res. 2016;10(8):QC04-07.

7. Tikkanen M, Nuutila M, Hiilesmaa V, Paavonen J, Ylikorkala O. Clinical presentation and risk factors of placental abruption. Acta Obstet Gynecol Scand. 2006;85(6):700-705.

8. Pariente G, Wiznitzer A, Sergienko R, Mazor M, Holcberg G, Sheiner E. Placental abruption: critical analysis of risk factors and perinatal outcomes. J Matern Fetal Neonatal Med. 2011;24(5):698-702.

9. Oyelese Y, Ananth CV. Placental abruption. Obstet Gynecol. 2006;108(4):1005-1016.

10. Mahendra G, Pukale RS, Vijayalakshmi S. Couvelaire uterua case report. IAIM. 2015;2:142-145. 